\title{
Knowledge Reconfiguration considering the distance of personal preference
}

\author{
JeongYon Shim \\ Division of General Studies, Computer Science, Kangnam University \\ San 6-2, Kugal-ri, Kihung-up, YongIn Si, KyeongKi Do, Korea, Tel: +82 312803 736, \\ mariashim@kangnam.ac.kr
}

\begin{abstract}
For the purpose of processing data efficiently in the huge data environment, a design of intelligent system based on the function of human brain is necessary. This paper describes how to reconstruct the efficient subject memory considering the personal preference from the objective facts. Conceptual modeling of new knowledge reconfiguration based on the common node connection from a different memory is proposed. The well formed structure of knowledge frame with special synonym list was designed for the efficient knowledge reconfiguration, and using this structure Knowledge retrieval mechanism was made to perform extracting the associated data.We applied this mechanism to the supposed virtual knowledge frame and tested.
\end{abstract}

\section{Introduction}

Generally it is known that everything is connected with others according to the interactive associations and has a dynamic connectionism in the world. Things are connected to other things according to their basic structure and common sense rules. However living creatures do not take the original form of all the information but select the important factors and information appropriate for surviving in the environment. The selected factors are reconstructed and adjusted in the inner frame. It is a very efficient mechanism for small living things to survive in the complicate circumstance. As they reconstruct the important information and functions very closely according to their individual interests, they can react to stimulus promptly and process the information efficiently in spite of small intellectual part compared to the amount of huge data in the environment. It is guessed that the memory formation of human brain is similar to this mechanism and memory is formed by reconfiguring the selected information from the outside according to the personal preference and characteristics. It is because he has a different memory and a different thinking mechanism that human being has his own thinking way. The structure of knowledge in human brain is not fixed but dynamically changes. New knowledge comes in and is stored and an unimportant data decay and are forgotten. The complexity lies not only in changing but also in many different memory frame. But human brain can always maintain the alive new knowledge in high degree of importance.During forming knowledge, the new data from the outside are reconstructed by being adjusted to 
his own knowledge structure. This reconfiguration function is an indispensable element for efficient memory management. It is also an very important function for preventing the redundancy and inconsistency problem of knowledge Base .

Many studies for solving redundancy and inconsistency problem have been made for many years in Database Researching area. But it is limited to standardizing the inconsistent concepts or terminologies[3,7].

Accordingly, in this paper we define Personal distance of knowledge frame and propose memory reconfiguration mechanism based on the personal distance for the efficient knowledge frame management. In this mechanism, an incoming inconsistent knowledge is reconfigured from the common node in the existing knowledge frame. A key point of this study is how to reconstruct the efficient subject memory considering the personal preference from the objective facts.

\section{Knowledge Reconfiguration in the memory}

\subsection{Knowledge Representation : Actual distance vs. Personal distance}

The structure of knowledge frame has a logical form of knowledge net according to their associations as shown in figure 1. This figure shows the reconfiguration of personal memory from the different sources. Actual knowledge net is structured by common sense knowledge and rules. But Personal Knowledge net is configured by various factors,i.e,personal preference, personal interest and the degree of stimuli from different Actual knowledge nets or new input cluster of knowledge.

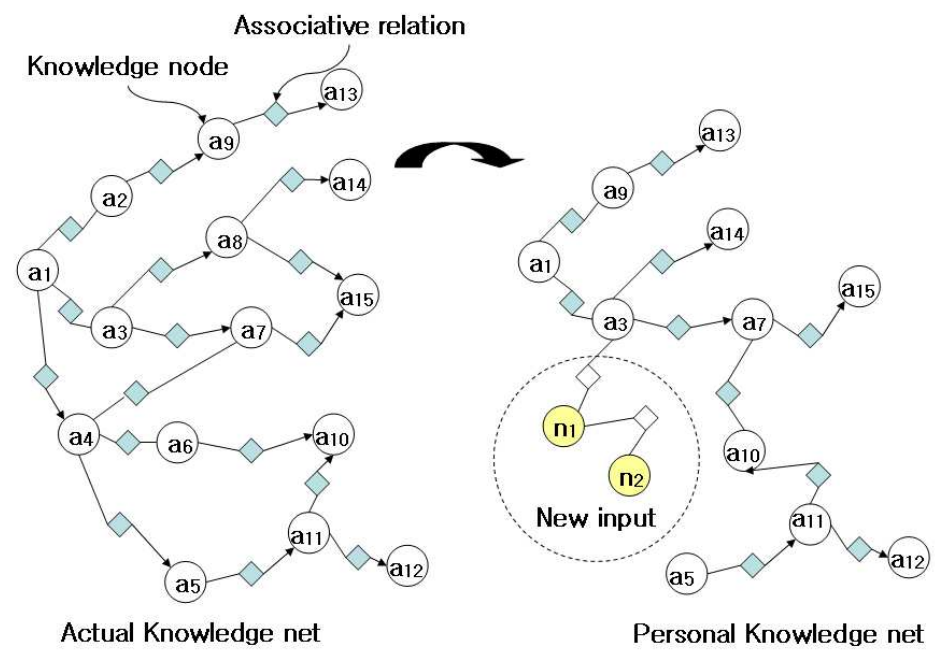

Fig. 1. Actual knowledge net vs. Personal knowledge net 
The basic Knowledge net consists of knowledge node and associative relation. Knowledge node has a keyword representing the name of the knowledge. Associative relation includes its associative linguistic relation name,associative degree and distance.

Associative degree $W_{i j}$ is conditional probability from $a_{1}$ to $a_{2}$ as following equation. It represents an associative strength between two nodes.

$$
W_{i j}=P\left(a_{i} \mid a_{j}\right)
$$

We define the distance between node $a_{1}$ and node $a_{2}$ in Actual Knowledge net as Actual distance, $d_{i j}$ which has a value of $[0,1]$. The subjective distance reconfigured in Personal Knowledge net is defined as Personal distance. Personal distance $d_{i j}^{\prime}$ is calculated by:

$$
d_{i j}^{\prime}=\frac{1}{1+e^{-\gamma}}, \gamma=\delta_{i j}+\Sigma d_{i k} * d_{k j}
$$

where $\delta_{i j}$ isavalueofpersonalpreference, $[0,1]$

\section{Knowledge Reconfiguration Mechanism}

As described in the previous section, memory Reconfiguration is a necessary function for maintaining the accurate and substantial knowledge frame. The incoming new knowledge and its associative relation should be estimated in Evaluation module for confidence. In Evaluation module, Confidence factor, $C_{i}$ is calculated using Importance factor, $I_{i}$, and frequency degree, $F_{i}$. Knowledge Reconfiguration module decides if it make this incoming new knowledge take part in the reconfiguration process with Confidence factor.

$$
F_{i}=\frac{1}{1+e^{-n}}
$$

Where $\mathrm{n}$ denotes frequency of access. Confidence factor is calculated by equation 4 .

$$
C_{i}=\frac{I_{i}+F_{i}}{2}
$$

\section{Memory Reconfiguration}

Memory reconfiguration mechanism starts to search the common nodes in the existing frame when a new knowledge frame comes in. If the common node is found, reconfiguring process centering around common node is made as shown in figure 2 .

However, there are several cases for reconfiguration during searching the correspondent nodes and relation in the existing knowledge frame. Accordingly, five cases are considered as follows.

Case $1:\left(a_{i}=a_{\text {newi }}, a_{j}=a_{n e w j}, R_{i j}=R_{\text {newij }}\right)$

In this case the weight of associative relation and Personal distance are adjusted by the equation because all the three terms are correspondent. 

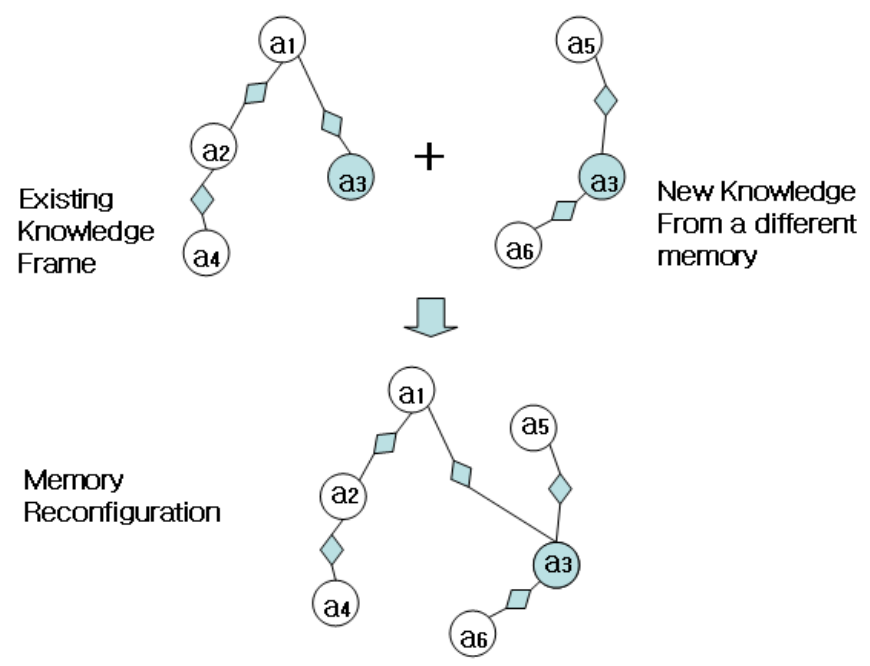

Fig. 2. Knowledge reconfiguration

$$
\begin{gathered}
K=W_{i j}+W_{n e w i j} \\
W_{i j}=\frac{1}{1+e^{-K}} \\
d_{i j}^{\prime}=\frac{1}{1+e^{-\gamma}}, \gamma=\delta_{i j}+\Sigma d_{i k} * d_{k j}
\end{gathered}
$$

Case $2:\left(a_{i}=a_{n e w i}, a_{j}=a_{n e w j}, R_{i j} \neq \mathbf{R}_{n e w i j}\right)$

The new associative relation link is created.

Case $3:\left(a_{i}=a_{n e w i}, a_{j} \neq \mathbf{a}_{n e w j}, R_{i j} \neq \mathbf{R}_{n e w i j}\right)$

The new associative relation link and knowledge node are created.

Case $4:\left(a_{i}=a_{\text {newi }}, a_{j} \neq \mathbf{a}_{\text {newj }}, R_{i j}=R_{\text {newij }}\right)$

In this case adjusting process is required because there is a conflict between the existing frame and incoming new knowledge frame. If the strength of associative relation between the existing nodes is greater than the strength of new one,the new frame is aborted. Otherwise, the links of the existing frame are deleted, new link is created and the new strength substitutes for the old one.

Case $5:\left(a_{i} \neq \mathbf{a}_{n e w i}, a_{j} \neq \mathbf{a}_{n e w j}, R_{i j} \neq \mathbf{R}_{n e w i j}\right)$

A new link is connected to the root node because there is no correspondent frame to the new frame in the existing memory.

When the new knowledge and associative relation selected in evaluation testing are propagated into the knowledge frame, Knowledge reconfiguration mechanism starts according to the following Algorithm 1.

Algorithm 1 : Knowledge Reconfiguration algorithm 
(I/O interface)

Step 1 : Input the new knowledge, associative degree and the importance of the knowledge $I_{i}$.

(Temporary memory)

Step 2 : Store on Temporary memory.

(Evaluation Module)

Step 3 : Calculate confidence degree.

$$
\begin{aligned}
F_{i} & =\frac{1}{1+e^{-n}} \\
C_{i} & =\frac{I_{i}+F_{i}}{2}
\end{aligned}
$$

Step $4: \operatorname{If}\left(C_{i} \geq \epsilon\right) \&(t \geq T)$

Then goto Forgetting module

Else goto Knowledge Reconfiguration module.

Forgetting module :

Move the input knowledge to the forgetting pool and remove it.

Knowledge Reconfiguration module :

\section{Step 4-1 :}

Search the representative name in the knowledge node and synonym list.

Step 4-2 :

Case $1\left(a_{i}=a_{n e w i}, a_{j}=a_{n e w j}, R_{i j}=R_{n e w i j}\right)$

Adjust the associative relation degree

$$
\begin{gathered}
K=W_{i j}+W_{n e w i j} \\
W_{i j}=\frac{1}{1+e^{-K}} \\
d_{i j}^{\prime}=\frac{1}{1+e^{-\gamma}}, \gamma=\delta_{i j}+\Sigma d_{i k} * d_{k j}
\end{gathered}
$$

Case $2\left(a_{i}=a_{\text {newi }}, a_{j}=a_{\text {newj }}, R_{i j} \neq \mathrm{R}_{\text {newij }}\right)$

Create the new associative relation

Case $3\left(a_{i}=a_{\text {newi }}, a_{j} \neq \mathrm{a}_{\text {newj }}, R_{i j} \neq \mathrm{R}_{\text {newij }}\right)$

Create the new link and node

Case $4\left(a_{i}=a_{\text {newi }}, a_{j} \neq \mathrm{a}_{\text {newj }}, R_{i j}=R_{\text {newij }}\right)$

the state of confliction

if $W_{i j} \geq \mathrm{W}_{\text {newij }}$

Then Abort the new knowledge pair

Else Delete the existing link and create the new link.

Case $5\left(a_{i} \neq \mathrm{a}_{\text {newi }}, a_{j} \neq \mathrm{a}_{\text {newj }}, R_{i j} \neq \mathrm{R}_{\text {newij }}\right)$

Create the associative relation and node connected to Root

Step 5: if knowledge pair $=\mathrm{t}$

Then goto Step 6

Else goto Step 1

Step 6: STOP. 


\section{Experiments}

We tested with the supposed virtual knowledge frame. Figure 3 shows an example used for experiments that test the reconfiguration with the selected knowledge frame from Actual knowledge net when the structures of actual knowledge net and existing Personal knowledge net are given.

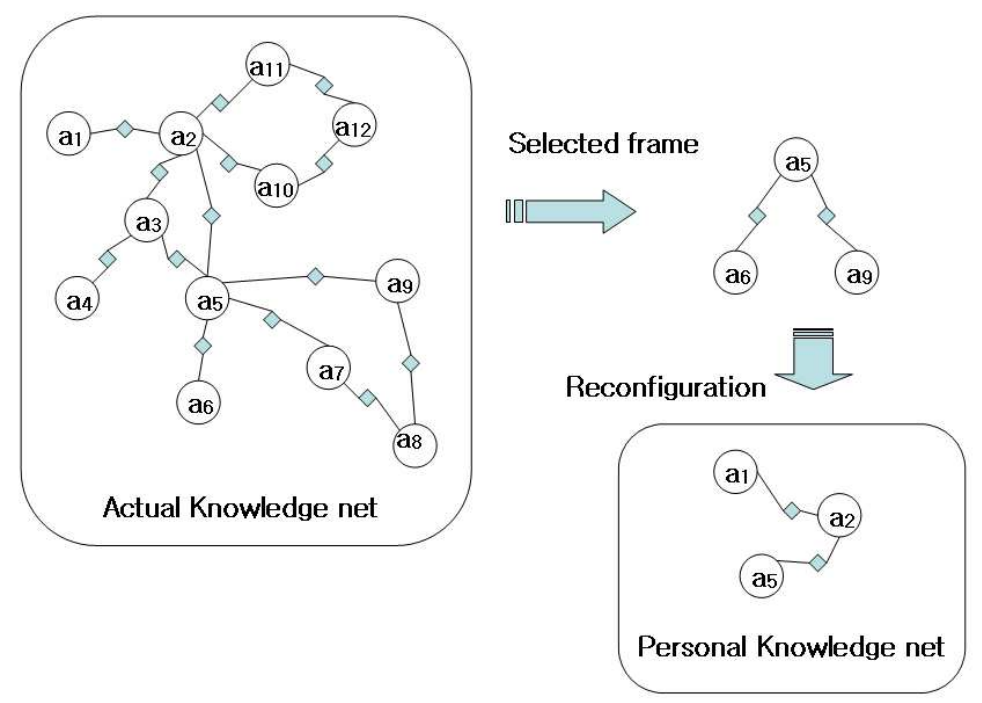

Fig. 3. example

Figure 4 depicts the initial data of node of Actual Knowledge net $a_{i j}$, Relation name $R_{i j}$, associative degree $W_{i j}$ and actual distance $d_{i j}$. The changed results by Knowledge Reconfiguration mechanism are shown in Figure 5. It shows the changed list of Personal Knowledge net including personal distance $d_{i j}^{\prime}$ after reconfiguration.

From the experiment, we can find that it reforms the knowledge frame and extracts the related data using this structure easily. It can solve redundancy and inconsistency problem of DB.

\section{Conclusion}

We proposed Conceptual model of new knowledge reconfiguration based on the common node connection from a different memory. We defined Personal distance of knowledge frame and propose memory reconfiguration mechanism based on the personal distance for the efficient knowledge frame management. In this 
* Actual Knowledge net

\begin{tabular}{|c|c|c|c|c|c|c|}
\hline node1 & node2 & & $-\mathbf{i j}$ & & $\mathbf{w}-\mathbf{i} \mathbf{j}$ & $\mathbf{d}-\mathbf{i} \mathbf{j}$ \\
\hline a1 & a2 & $\mathbf{R}$ & & 2 & 5.89 & 0.36 \\
\hline a2 & a3 & $\mathbf{R}$ & 2 & 3 & 0.70 & 0.36 \\
\hline a2 & a5 & $\mathbf{R}$ & & 5 & 0.60 & 0.36 \\
\hline a2 & a15 & $\mathbf{R}$ & 2 & 15 & 5.20 & 5.46 \\
\hline a2 & a11 & $\mathbf{R}$ & 2 & 11 & 0.20 & 0.36 \\
\hline a3 & a4 & $\mathbf{R}$ & 3 & 4 & 0.90 & 0.46 \\
\hline a3 & a5 & $\mathbf{R}$ & 35 & 5 & 0.40 & 0.36 \\
\hline a4 & Nill & $\mathbf{R}$ & 4 & 4 & 5.05 & 5.05 \\
\hline a5 & a6 & $\mathbf{R}$ & 5 & 6 & 0.42 & 0.36 \\
\hline a5 & $a 7$ & $\mathbf{R}$ & 57 & 7 & 0.50 & 0.26 \\
\hline a5 & a9 & $\mathbf{R}$ & 5 & 9 & 0.60 & 0.36 \\
\hline a6 & Nill & $\mathbf{R}$ & 6 & 6 & 5.00 & 0.01 \\
\hline a7 & a8 & $\mathbf{R}$ & 78 & 8 & 0.70 & 0.36 \\
\hline a8 & Nill & $\mathbf{R}$ & 88 & 8 & 0.00 & 0.01 \\
\hline a9 & a8 & $\mathbf{R}$ & 98 & 8 & 0.20 & 0.35 \\
\hline a10 & a12 & $\mathbf{R}$ & 10 & 12 & 0.10 & 6.36 \\
\hline a11 & a12 & $\mathbf{R}$ & 11 & 12 & 5.40 & 0.36 \\
\hline a12 & Nill & $\mathbf{R}$ & 12 & 12 & 5.00 & 6. 015 \\
\hline
\end{tabular}

Fig. 4. Actual Knowledge net

mechanism, an incoming inconsistent knowledge is reconfigured from the common node in the existing knowledge frame. A key point of this study is how to reconstruct the efficient subject memory considering the personal preference from the objective facts.

As a result of testing, we could find that it can reform the knowledge frame and extract the related data using this structure easily. It could solve redundancy and inconsistency problem. This mechanism can be applied to construct personal memory structure and personal preference can be calculated by strength of associative relations. Personal preference is a very important information for providing service considering personal characteristics in many areas of E-commerce system,Database management and etc..

\section{References}

1. E. Bruce Goldstein Sensation and Perception BROOKS/COLE.

2. John R. Anderson Learning and Memory Prentice Hall.

3. R.A .Frost Introduction to Knowledge Base Systems WilliamCollins Sons Co. Ltd..

4. Jeong-Yon Shim, Chong-Sun Hwang Data Extraction from Associative Matrix based on Selective learning system IJCNN'99, Washongton D.C.

5. Jeong-Yon Shim, Chong-Sun Hwang Intelligent Hierarchical Associative Knowledge Learning System with Knowledge acquisition and Extraction , ICONIP2000, Deajeon, Korea. 
* Initial state of Personal net

\begin{tabular}{|c|c|c|c|c|c|}
\hline node1 & node2 & $\mathbf{R}-\mathbf{i} \mathbf{j}$ & $\mathbf{W}-\mathbf{i} \mathbf{j}$ & $\mathbf{d}-\mathbf{i} \mathbf{j}$ & $d^{\prime}-i j$ \\
\hline 1 & a & R 12 & 9 & 1.30 & \\
\hline a2 & a5 & R 25 & 0.60 & 0.30 & 0.987690 \\
\hline a5 & Nill & R 55 & 0.00 & 0.00 & 0. 000000 \\
\hline
\end{tabular}

* Reconfiguration of Personal net

\begin{tabular}{|c|c|c|c|c|c|c|}
\hline node1 & node2 & $\mathbf{R}-\mathbf{i}$ & & $\mathbf{W} \mathbf{i} \mathbf{j}$ & $\mathbf{d}-\mathbf{i} \mathbf{j}$ & $d^{\prime}-\mathbf{i} \mathbf{j}$ \\
\hline a1 & a2 & R 1 & 2 & 0.89 & 0.35 & 0.789045 \\
\hline a2 & a5 & R 2 & 5 & 0.65 & 0.35 & 0.987690 \\
\hline a2 & a10 & R 2 & 10 & 0.20 & 0.40 & 0.769876 \\
\hline a5 & a6 & R 5 & 6 & 0.42 & 0.30 & 0.678590 \\
\hline a5 & a9 & R 5 & 9 & 0.65 & 0.35 & 0.876795 \\
\hline a6 & Nill & R 6 & 6 & 0.50 & 5. 50 & อ. 595955 \\
\hline a9 & Nill & R 8 & 8 & 0.05 & 0.05 & 5. 509050 \\
\hline
\end{tabular}

Fig. 5. The list of Personal net after reconfiguration

6. Jean-Marc Adamo Data Mining for Association rules and sequential patterns , Springer.

7. Adrian A. Hopgood Knowledge based systems for engineers ans Scientist ,CRC press. 